\title{
NEAR-IDEMPOTENTS, NEAR-NILPOTENTS AND STABILITY OF CRITICAL POINTS FOR RICCATI EQUATIONS
}

\author{
Borut Zalar and Matej Mencinger* \\ University of Maribor, Slovenia
}

\begin{abstract}
The paper introduces two algebraic concepts, near-idempotents and near-nilpotents associated to subspaces $\mathcal{N}$ of critical points, which can be used to re-phrase a theorem due to Boujemaa, El Qotbi and Rouiouih on stability for the Ricatti equation, $\dot{x}=x(t)^{2}$, associated to algebra $\mathcal{A} \approx \mathbb{R}^{d}$. Using this concepts their result corresponds to the case $\operatorname{dim} \mathcal{N}=1$.

Our main results are a generalization of the above mentioned theorem to $\mathcal{N}$ of arbitrary dimension and a counter-example which shows, even in the general setting, that the essential condition that critical points must be eigenvectors of a suitable multiplication operator cannot be omitted from the formulation due to Boujemaa et al.
\end{abstract}

\section{INTRODUCTION}

This research is a part of our long-term aim to classify, up to a linear change of coordinates, all autonomous homogeneous quadratic systems of ODEs in $\mathbb{R}^{n}$ with origin as a stable critical point. We intend to use a ringtheoretic approach, pioneered by Markus, which is to classify commutative algebras of given dimension, up to isomorphisms, and determine which algebras correspond to stable systems. This task (to classify all finite-dimensional commutative non-associative algebras) seems impossible, so our strategy to classify all systems with stable origin is to first rule out large families with certain algebraic properties which forces the corresponding system to behave unstable near the origin.

2010 Mathematics Subject Classification. 34A34, 17A99.

Key words and phrases. Quadratic differential systems, non-associative algebra, singular points, stability, near-nilpotent, near-idempotent.

${ }^{*}$ Corresponding author: matej.mencinger@um.si. 
It is well-known and almost trivial to see that the presence of a nontrivial idempotent in the algebra makes the corresponding Riccati equation non-stable in the neighborhood of the origin. This eliminates quite a lot of algebras, but even in 2-dimensional case, if we try to establish an alternative (Markus-type) approach to the classical theory of planar stability, we end up with 10 (one-parametric families of) non-isomorphic algebras which do not contain idempotents. Even in 3-dimensional case the number of nonisomorphic classes of idempotent-free algebras would increase significantly, so we seek further conditions which would imply instability of origin.

In this paper we define a concept which we call "near-idempotent". Such elements exist even in algebras which do not contain idempotents and under a suitable condition they imply that the origin of the corresponding system of ODEs cannot be stable. In this way we hope to eliminate enough classes of algebras, to get a manageable list of non-isomorphic 3-dimensional algebras in order to classify all autonomous quadratic systems of ODEs with stable origin in $\mathbb{R}^{3}$, which is still a work in progress.

Let $Q: \mathbb{R}^{d} \rightarrow \mathbb{R}^{d}$ be a homogeneous form of degree two, i.e.

$$
Q(\alpha \vec{x})=\alpha^{2} Q(\vec{x}) \quad \forall \alpha \in \mathbb{R}, \quad \forall \vec{x} \in \mathbb{R}^{d} .
$$

An autonomous homogeneous polynomial systems of ODEs, defined by

$$
\vec{x}^{\prime}=\frac{d \vec{x}}{d t}=Q(\vec{x}),
$$

where the vector function $\vec{x}$ is defined on some real interval, is called a quadratic system.

The idea to connect the study of quadratic systems (1.2) with methods from nonassociative algebra originates to Markus ([17]). To every quadratic system (1.2) we can associate its (Markus) algebra $\mathcal{A}=\left(\mathbb{R}^{n}, \cdot\right)$, where the algebra multiplication $\cdot$ is defined by

$$
\vec{x} \cdot \vec{y}=\frac{1}{2}(Q(\vec{x}+\vec{y})-Q(\vec{x})-Q(\vec{y})) .
$$

Thus, in algebra $\mathcal{A}$, system (1.2) becomes a Riccati equation $\vec{x}^{\prime}=\vec{x} \cdot \vec{x}=\vec{x}^{2}$. This idea was considered by many authors (e.g. [5,6,3,10-12,14,2,19-21,23-25] to mention just few) and is applicable also for partial differential equations ([13]) and even for difference systems $([22,15])$. Note that the process called homogenization ([26, p. 22]) transforms any Riccati equation with constant coefficients into the form (1.3). The qualitative theory of quadratic homogeneous planar systems of ODEs is well-known (see $[1,7-9,16,18]$ for classical treatment and [19] for treatment of stability with an algebraic approach).

Obviously $\vec{x}=\overrightarrow{0} \in \mathbb{R}^{d}$ is a nonhyperbolic (even totally degenerated) singular point of (1.2) for every $d \in \mathbb{N}$. It is obvious that non-zero singular points of (1.2) correspond to nilpotents $\vec{n}$ of rank two (i.e. special nonzero 
elements $\vec{n}$ defined by $\vec{n} \cdot \vec{n}=\overrightarrow{0}$ ) of the algebra. Throughout this paper we consider the stability of singular points in the classical sense of Lyapunov.

It is also well-known that any nonzero idempotent in $\mathcal{A}$ (element $\vec{p}$ such that $\vec{p} \cdot \vec{p}=\vec{p}$ holds) implies the existence of ray solutions of (1.2), yielding unstable dynamics of the origin. The solutions of (1.2) lying on a line $\mathbb{R} \vec{p}$ are called blow-up solutions $([11,12])$.

The problem of the stability of the origin was completely solved for 2dimensional case in [19]. For 3-dimensional case only partial results are known. In [23, Theorem 2.1] we initiated the study of stability of other (non-zero) singular points. Denote the $\lambda$-space of a nonzero element $\vec{u}$ by

$$
\mathcal{A}_{\lambda}(\vec{u})=\{\vec{x} \in \mathcal{A} ; \vec{u} \cdot \vec{x}=\lambda \vec{x}\} .
$$

If $\mathcal{A}_{\lambda}(\vec{u}) \neq\{0\}$, we call it an eigenspace of $\vec{u}$. Since all such $\lambda$ are eigenvalues of the linear map $\vec{x} \rightarrow \vec{u} \cdot \vec{x}$, the maximal number of $\lambda$-eigenspaces is smaller than the dimension $d$. We proved in [23, Theorem 2.1] that a nonzero nilpotent $\vec{n}$ of order two, which is included in one of the eigenspaces of a nonzero idempotent $\vec{p}$, i.e. $\vec{n} \in A_{\lambda}(\vec{p})$, represent a nonstable singular point of (1.2). Note that this means that $\mathbb{R} \vec{n}=\{\vec{x} \in \mathcal{A} ; \vec{x}=a \vec{n}\} \subset A_{\lambda}(\vec{p})$, therefore the line $\mathbb{R} \vec{n}$ consists entirely of nonstable singular points for the Riccati equation.

Our result was improved by Boujemaa, El Qotbi and Rouiouih ([4]) in two directions. They first proved that the above mentioned result remains true even when $\vec{p}$ is not necessarily an idempotent but an element satisfying a weaker algebraic condition, and then proceed to completely solve the problem when a singular point (i.e. nilpotent in the corresponding Markus algebra) of 2-dimensional quadratic system is stable.

In this paper we define a general algebraic framework in which the first main result of [4] can be interpreted as a special case where the subspace of singular points is 1-dimensional. The second section will provide a clarification of 2-dimensional case in this new terminology.

In the third section we prove our main result which is a natural extension of Boujemaa-El Qotbi-Rouiouih theorem to general subspaces of singular points and provide a counter-example in $\mathbb{R}^{3}$, in which the subspace of singular points is 2-dimensional, whose purpose is to show that the additional assumption of [23, Theorem 2.1] and [4], i.e. that singular points must belong to some eigenspaces of suitable algebraic elements, cannot be omitted.

\section{Definitions AND REINTERPREtation OF 2-Dimensional CASE}

Let $\mathcal{A}$ be a commutative real algebra and $\mathcal{N} \subset \mathcal{A}$ a subspace spanned by a set of nilpotent elements, i.e. there exists a basis $\vec{n}_{1}, \ldots, \vec{n}_{k}$ for $\mathcal{N}$, such that $\vec{n}_{i}^{2}=0$ for all $i=1,2, \ldots, k$. An element $\vec{u} \in \mathcal{A} \backslash \mathcal{N}$ will be called a 
near-nilpotent associated to $\mathcal{N}$ if

$$
\vec{u}^{2}=\sum_{i=1}^{k} \lambda_{i} \vec{n}_{i},
$$

where all $\lambda_{i} \in \mathbb{R}$ are nonzero.

An element $\vec{u} \in \mathcal{A} \backslash \mathcal{N}$ will be called a near-idempotent associated to $\mathcal{N}$ if

$$
\vec{u}^{2}-\vec{u}=\sum_{i=1}^{k} \lambda_{i} \vec{n}_{i},
$$

where all $\lambda_{i} \in \mathbb{R}$ are nonzero.

The largest possible number $k$ from equation (2.1) and/or (2.2) will be the rank of $\vec{u}$. Note that the above definitions imply that $\vec{u}^{2}$ is always a nonzero element.

Eigenspaces of $\vec{u}$ are those subspaces of the form (1.4) which are nontrivial, i.e. contain non-zero elements of $\mathcal{A}$. In the present terminology, Boujemaa-El Qotbi-Rouiouih theorem ([4, Th. 2.1]) can be rewritten as follows.

Proposition 2.1. Let $\mathcal{A}$ be a commutative real algebra of finite dimension and $\vec{u} \in \mathcal{A}$ either a near-idempotent or near-nilpotent of rank 1, associated to a subspace $\mathcal{N}=\mathbb{R} \vec{n}_{0}$, where $\vec{n}_{0}$ is a nonzero nilpotent. If $\mathcal{N}$ is included in one of the eigenspaces of $\vec{u}$, then every $\vec{n} \in \mathcal{N}$ is a nonstable singular point of the Riccati equation $\vec{x}^{\prime}=\vec{x}^{2}$ associated with $\mathcal{A}$.

In the sequel we combine some of our previous work and Theorem 2.2 of [4], in order to obtain some interesting observations for the smallest possible case, i.e. $\operatorname{dim}(\mathcal{A})=2$ and $\operatorname{dim}(\mathcal{N})=1$.

Proposition 2.2. A two-dimensional real commutative algebra $\mathcal{A}$ that does not contain idempotents, near-idempotents nor near-nilpotents has a trivial multiplication, i.e. $\vec{x} \cdot \vec{y}=0$ for all $\vec{x}, \vec{y} \in \mathcal{A}$.

Proof. From the proof of [19, Theorem 3] it follows that every twodimensional algebra which does not contain an idempotent is isomorphic to one of the following six possibilities, which can be treated separately:

(i) $[19$, Theorem 3 , case 1] the algebra with trivial multiplication, in which case there is nothing to prove;

(ii) $\left[19\right.$, Theorem 3 , case 2] an algebra $\mathcal{A}=\mathbb{R} \vec{n} \oplus \mathbb{R} \vec{e}$ such that $\vec{n}^{2}=0$, $\vec{e}^{2}=0, \vec{n} \cdot \vec{e}=\vec{e}$; in this case we can take $\mathcal{N}=\mathbb{R} \vec{n}$ and $\vec{u}=\frac{1}{2} \vec{n}+\vec{e} \notin \mathcal{N}$. It is easy to see that $\vec{u}^{2}-\vec{u}=\frac{1}{2} \vec{n} \in \mathcal{N}$, i.e. $\vec{u}$ is a near-idempotent associated with $\mathcal{N}$;

(iii) $\left[19\right.$, Theorem 3 , case 5] an algebra $\mathcal{A}=\mathbb{R} \vec{n} \oplus \mathbb{R} \vec{e}$ such that $\vec{n}^{2}=0$, $\vec{e}^{2}=\vec{n}, \vec{n} \cdot \vec{e}=\overrightarrow{0} ;$ in this case we again define $\mathcal{N}=\mathbb{R} \vec{n}$. Since $\vec{e} \notin \mathcal{N}$ and $\vec{e}^{2}=1 \cdot \vec{n}$, by defining $\vec{u}=\vec{e}$ we obviously obtain a near-nilpotent associated with $\mathcal{N}$ 
(iv) $\left[19\right.$, Theorem 3 , case 7] an algebra $\mathcal{A}=\mathbb{R} \vec{n} \oplus \mathbb{R} \vec{e}$ such that $\vec{n}^{2}=0$, $\vec{e}^{2}=\vec{n}+2 \vec{e}, \vec{n} \cdot \vec{e}=\vec{n}$; in this case for $\mathcal{N}=\mathbb{R} \vec{n}$ and $\vec{u}=\frac{1}{2} \vec{e} \notin \mathcal{N}$ we obtain $\vec{u}^{2}-\vec{u}=\frac{1}{4}(\vec{n}+2 \vec{e})-\frac{1}{2} \vec{e}=\frac{1}{4} \vec{n} \in \mathcal{N}$ therefore $\vec{u}$ is a near-idempotent associated with $\mathcal{N}$;

(v) $\left[19\right.$, Theorem 3, case 9] an algebra $\mathcal{A}=\mathbb{R} \vec{n} \oplus \mathbb{R} \vec{e}$ such that $\vec{n}^{2}=0$, $\vec{e}^{2}=-\vec{n}, \vec{n} \cdot \vec{e}=\vec{e}$; in this case for $\mathcal{N}=\mathbb{R} \vec{n}$ and $\vec{u}=\vec{e}+\frac{1}{2} \vec{n} \notin \mathcal{N}$ we obtain

$$
\begin{aligned}
\vec{u}^{2}-\vec{u} & =\vec{e}^{2}+\vec{e} \cdot \vec{n}-\vec{e}-\frac{1}{2} \vec{n} \\
& =-\vec{n}+\vec{e}-\vec{e}-\frac{1}{2} \vec{n}=-\frac{3}{2} \vec{n} \in \mathcal{N}
\end{aligned}
$$

therefore $\vec{u}$ is a near-idempotent associated with $\mathcal{N}$;

(vi) [19, Theorem 3, case 10] an algebra $\mathcal{A}=\mathbb{R} \vec{n} \oplus \mathbb{R} \vec{e}$ such that $\vec{n}^{2}=0$, $\vec{e}^{2}=\kappa \vec{n}+\vec{e}$, where $\kappa<-\frac{1}{8}$, and $\vec{n} \cdot \vec{e}=\vec{e}$; in this case for $\mathcal{N}=\mathbb{R} \vec{n}$ and $\vec{u}=\vec{e} \notin \mathcal{N}$ we obtain $\vec{u}^{2}-\vec{u}=\kappa \vec{n}+\vec{e}-\vec{e}=\kappa \vec{n} \in \mathcal{N}$, since $\kappa \neq 0$, therefore $\vec{u}$ is a near-idempotent associated with $\mathcal{N}$.

The above analysis shows that in all cases (i)-(vi) we obtain at least one desired element, which concludes the proof.

Proposition 2.3. A two-dimensional real commutative algebra which does not contain neither idempotents nor near-idempotents is isomorphic to one of the following algebras

\begin{tabular}{|c||c|c|}
\hline$\cdot$ & $\vec{n}$ & $\vec{e}$ \\
\hline \hline$\vec{n}$ & $\overrightarrow{0}$ & $\overrightarrow{0}$ \\
\hline$\vec{e}$ & $\overrightarrow{0}$ & $\overrightarrow{0}$ \\
\hline
\end{tabular}

or

(B)

\begin{tabular}{|c||c|c|}
\hline$\cdot$ & $\vec{n}$ & $\vec{e}$ \\
\hline$\vec{n}$ & $\overrightarrow{0}$ & $\overrightarrow{0}$ \\
\hline$\vec{e}$ & $\overrightarrow{0}$ & $\vec{n}$ \\
\hline
\end{tabular}

PROOF. In the previous proof we saw that among six non-isomorphic types of algebras which do not contain an idempotent, types (ii), (iv), (v) and (vi) contain a near-idempotent. It remains to prove that algebras of type (iii), while containing near-nilpotent element, do not contain near-idempotents. Let $\alpha, \beta \in \mathbb{R}$ be arbitrary and $\vec{x}=\alpha \vec{n}+\beta \vec{e}$. From the multiplication table (B) we obtain $\vec{x}^{2}=\beta^{2} \vec{n}$, which can be zero only if $\beta=0$, i.e. $\mathbb{R} \vec{n}$ is the only possibility for $\mathcal{N}$ in definition (2.2). For $\vec{u}=\gamma \vec{n}+\delta \vec{e}$, in order for $\vec{u}$ to be a near-idempotent associated with $\mathcal{N}$, we must have $\delta \neq 0$ and

$$
\begin{aligned}
\vec{u}^{2}-\vec{u} & =(\gamma \vec{n}+\delta \vec{e})^{2}-(\gamma \vec{n}+\delta \vec{e}) \\
& =\delta^{2} \vec{e}^{2}-\gamma \vec{n}-\delta \vec{e} \\
& =\delta^{2} \vec{n}-\gamma \vec{n}-\delta \vec{e} \in \mathbb{R} \vec{n},
\end{aligned}
$$

which is impossible.

COROLlary 2.4. The additional assumption, about $\mathcal{N}$ being an eigenspace of $\vec{u}$, cannot be removed from Proposition 2.1. 
Proof. Consider the algebra (v) from the proof of Proposition 2.2. From $\vec{n}^{2}=0, \vec{n} \cdot \vec{e}=\vec{e}, \vec{e}^{2}=-\vec{n}$ and $\vec{u}=\vec{e}+\frac{1}{2} \vec{n}$ we compute the multiplication table in terms of $\vec{n}$ and $\vec{u}$ :

\begin{tabular}{|c||c|c|}
\hline$\cdot$ & $\vec{n}$ & $\vec{u}$ \\
\hline \hline$\vec{n}$ & $\overrightarrow{0}$ & $\frac{1}{2} \vec{u}-\frac{5}{4} \vec{n}$ \\
\hline$\vec{u}$ & $\frac{1}{2} \vec{u}-\frac{5}{4} \vec{n}$ & $\vec{u}-\frac{3}{2} \vec{n}$ \\
\hline
\end{tabular}

From this table it is obvious that $\vec{u} \cdot \vec{n}$ is not a scalar multiple of $\vec{n}$, therefore $\mathcal{N}=\mathbb{R} \vec{n}$ is not an eigenspace of $\vec{u}$. In Theorem 2.2 of [4] it was proved that for systems of the form

\begin{tabular}{|c||c|c|}
\hline$\cdot$ & $\vec{u}$ & $\vec{n}$ \\
\hline \hline$\vec{u}$ & $\delta \vec{u}+\gamma \vec{n}$ & $\beta \vec{u}+\alpha \vec{n}$ \\
\hline$\vec{n}$ & $\beta \vec{u}+\alpha \vec{n}$ & $\overrightarrow{0}$ \\
\hline
\end{tabular}

the conditions $\alpha \beta \neq 0$ and $\beta>0$ imply that the critical point $a \vec{n}$ is stable for every negative number $a$. If we apply this theorem to parameters $\alpha=-\frac{5}{4}$, $\beta=\frac{1}{2}, \delta=1$ and $\gamma=-\frac{3}{2}$, it follows that $\mathcal{N}$ contains stable points.

\section{Main RESUlt AND a COUNTER-EXAMPle}

There are several possibilities how to generalize Boujemaa-El QotbiRouiouih theorem. Obviously, since their theorem treats elements of rank 1, we want to consider the general rank, but it is not immediately clear what additional condition to impose on the subspace of critical points. If $\operatorname{dim} \mathcal{N}=1$, it is obvious that $\mathcal{N}=\mathbb{R} \vec{n}$ is both a nil-algebra $\left(\vec{n}_{1} \cdot \vec{n}_{2}=(\alpha \vec{n}) \cdot(\beta \vec{n})=\alpha \beta \vec{n}^{2}=0\right)$ and a subalgebra of $\mathcal{A}$. We have therefore three progressing possibilities: (i) impose no additional assumption for $\mathcal{N}$, (ii) assume that $\mathcal{N}$ is a subalgebra of $\mathcal{A}$, i.e. $\mathcal{N} \cdot \mathcal{N} \subset \mathcal{N}$ or (iii) assume $\mathcal{N} \cdot \mathcal{N}=0$. The first two are very difficult, but we believe that under (i) the Boujemaa-El Qotbi-Rouiouih-type theorem for higher ranks is not true. The assumption (ii) is not easy for the case of nearnilpotents even if $\operatorname{dim} \mathcal{A}=3$ and $\operatorname{dim} \mathcal{N}=2$, which is still work in progress. At present we have no idea how to treat the general case. The assumption (iii) is sufficient to provide a meaningful generalization in the following way.

THEOREM 3.1. Let $\mathcal{A}$ be a commutative real algebra of dimension greater then $k$ and $\vec{n}_{1}, \ldots, \vec{n}_{k}$ nonzero linearly independent nilpotents of rank two. Denote $\mathcal{N}=\operatorname{span}\left(\vec{n}_{1}, \ldots, \vec{n}_{k}\right)$ and assume $\vec{n}_{i} \cdot \vec{n}_{j}=\overrightarrow{0}$ for all $1 \leq i, j \leq k$. Let $\vec{u} \in \mathcal{A} \backslash \mathcal{N}$ be a near-nilpotent or a near-idempotent associated with $\mathcal{N}$. If $\vec{n}_{i} \in A_{\lambda_{i}}(\vec{u})$, for all $1 \leq i \leq k$ and some set of real numbers $\lambda_{1}, \ldots, \lambda_{k}$, then any $\vec{n} \in \mathcal{N}$ is a nonstable singular point of the Riccati equation $\vec{x}^{\prime}=\vec{x}^{2}$.

Proof. In order to prove that some singular point $\vec{n}$ is not stable in the sense of Lyapunov, it is sufficient to show that every neighborhood of $\vec{n}$ contains a point such that the particular solution with this point as its initial 
value can lead far from $\vec{n}$. We can decompose a real space $\mathcal{A}$ into a sum of subspaces $\mathcal{A}=\mathbb{R} \vec{u} \oplus \mathcal{N} \oplus \mathcal{W}$, where $W$ represents remaining coordinates, which cannot be expressed in terms of $\vec{u}, \vec{n}_{1}, \ldots, \vec{n}_{k}$. Since $\vec{n} \in \mathcal{N}$, all its neighborhoods contain some points whose coordinates $w \in \mathcal{W}$ equal to zero. Since $\mathcal{B}=\mathbb{R} \vec{u} \oplus \mathcal{N}$ is a subalgebra of $\mathcal{A}$, the solution $\vec{x}(t)$ with the initial condition $\alpha_{0} \vec{u}+\sum \alpha_{i} \vec{n}_{i}$, will have its $w$ - coordinates equal to zero for all times $t$. Thus, we can restrict our attention to $\mathcal{B}$.

According to the assumptions of the theorem, there exists a basis for which $\mathcal{B}$ assumes the following multiplication table:

\begin{tabular}{|c||c|c|c|c|}
\hline$\cdot$ & $\vec{n}_{1}$ & $\cdots$ & $\vec{n}_{k}$ & $\vec{u}$ \\
\hline \hline$\vec{n}_{1}$ & $\overrightarrow{0}$ & $\cdots$ & $\overrightarrow{0}$ & $\lambda_{1} \vec{n}_{1}$ \\
\hline$\vdots$ & $\vdots$ & $\ddots$ & $\vdots$ & $\vdots$ \\
\hline$\vec{n}_{k}$ & $\overrightarrow{0}$ & $\cdots$ & $\overrightarrow{0}$ & $\lambda_{k} \vec{n}_{k}$ \\
\hline$\vec{u}$ & $\lambda_{1} \vec{n}_{1}$ & $\cdots$ & $\lambda_{k} \vec{n}_{k}$ & $\delta \vec{u}+\sum_{i=1}^{k} \gamma_{i} \vec{n}_{i}$ \\
\hline
\end{tabular}

where $\delta, \lambda_{1}, \gamma_{1}, \ldots, \lambda_{k}, \gamma_{k} \in \mathbb{R}$. By setting

$$
\vec{x}(t)=\sum_{i=1}^{k} g_{i}(t) \vec{n}_{i}+f(t) \vec{u}
$$

where $f, g_{1}, \ldots, g_{k}$ are some differentiable real functions, the corresponding quadratic system assumes the coordinate form

$$
\begin{aligned}
g_{1}^{\prime} & =\gamma_{1} f^{2}+2 \lambda_{1} f g_{1} \\
& \vdots \\
g_{k}^{\prime} & =\gamma_{k} f^{2}+2 \lambda_{k} f g_{k} \\
f^{\prime} & =\delta f^{2}
\end{aligned}
$$

Let $\vec{n}=\sum \alpha_{i} \vec{n}_{i} \in \mathcal{N}$ be an arbitrary nilpotent. Every neighborhood of $\vec{n}$ contains elements of the form $\varepsilon \vec{u}+\vec{n}$, where $\varepsilon$ is a nonzero number. Let $\vec{x}(t)$ be the solution of $\vec{x}^{\prime}=\vec{x}^{2}$ with initial condition $\vec{x}(0)=\varepsilon \vec{u}+\vec{n}$, i.e. $f(0)=\varepsilon$ and $g_{i}(0)=\alpha_{i}$. Suppose first $\delta \neq 0$. We choose the sign of $\varepsilon$ in such a way, that the number $\delta \varepsilon$ becomes positive. The last component of the solution obviously equals

$$
f(t)=\frac{\varepsilon}{1-\varepsilon \delta t},
$$

which is defined for any $0 \leq t<\frac{1}{\varepsilon \delta}$ and blows up in finite time $t_{\infty}=\frac{1}{\varepsilon \delta}$. This proves that $\vec{n}$ is not stable if $\delta \neq 0$. 
Now we suppose that $\delta=0$ and consider the same initial condition $\vec{x}(0)=$ $\varepsilon \vec{u}+\vec{n}$. The corresponding system of ODEs then becomes

$$
\begin{aligned}
g_{1}^{\prime} & =\gamma_{1} f^{2}+2 \lambda_{1} f g_{1}, \\
& \vdots \\
g_{k}^{\prime} & =\gamma_{k} f^{2}+2 \lambda_{k} f g_{k}, \\
f^{\prime} & =0 .
\end{aligned}
$$

The initial conditions $g_{1}(0)=\alpha_{1}, g_{2}(0)=\alpha_{2}, \ldots, g_{k}(0)=\alpha_{k}, f(0)=\varepsilon$ first implies the constant solution $f(t)=\varepsilon$ for the last component and consequently the following system of first order linear equations

$$
g_{i}^{\prime}=\gamma_{i} \varepsilon^{2}+2 \lambda_{i} \varepsilon g_{i} ; \quad 1 \leq i \leq k .
$$

Note that $\gamma_{i} \neq 0$, for all $1 \leq i \leq k$, by assumption. The solution to (3.3) can be explicitly computed. The results of those computations can be summarized as

$$
g_{i}(t)=\left\{\begin{array}{lll}
\left(\alpha_{i}+\frac{\varepsilon \gamma_{i}}{2 \lambda i}\right) e^{2 \lambda_{i} \varepsilon t}-\frac{\varepsilon \gamma_{i}}{2 \lambda_{i}} & \text { if } & \alpha_{i} \neq 0 \text { and } \lambda_{i} \neq 0 \\
\frac{\gamma_{i} \varepsilon}{2 \lambda_{i}}\left(e^{2 \lambda_{i} \varepsilon t}-1\right) & \text { if } & \alpha_{i}=0 \text { and } \lambda_{i} \neq 0 \\
\alpha_{i}+t \varepsilon^{2} \gamma_{i}^{2} & \text { if } & \alpha_{i} \neq 0 \text { and } \lambda_{i}=0 \\
t \varepsilon^{2} \gamma_{i}^{2} & \text { if } & \alpha_{i}=0 \text { and } \lambda_{i}=0
\end{array}\right.
$$

These formulas obviously imply a blow-up solution (i.e. $\lim _{t \rightarrow \infty}\left|g_{i}(t)\right|=\infty$ ) for any instance of parameter values, since $\gamma_{i} \neq 0$ and we can choose $\varepsilon$ to be either positive or negative number, which in turn implies that the singular point $\vec{n}$ in not stable.

As our final result we will show that the condition that all base nilpotents belong to some eigenspace of a near-idempotent $\vec{u}$ cannot be omitted in Theorem 3.1. We will construct an example with $\mathcal{A}=\mathbb{R} \vec{u} \oplus \mathbb{R} \vec{n}_{1} \oplus \mathbb{R} \vec{n}_{2}$ and $\mathcal{N}=\mathbb{R} \vec{n}_{1} \oplus \mathbb{R} \vec{n}_{2}$, such that one base nilpotent belongs to some eigenspace of $\vec{u}$, the other base nilpotent does not belong to any eigenspace of $\vec{u}$, and $\mathcal{N}$ contains at least one stable singular point.

More precisely, we shall prove that the origin is a stable point of the system considered below. It seems that the question whether $\mathcal{N}$ contains some additional stable points is not a trivial one and we are not able to answer it at present.

Proposition 3.2. Consider the Riccati equation associated to a 3dimensional algebra with the multiplication table

\begin{tabular}{|c||c|c|c|}
\hline$\cdot$ & $\vec{n}_{1}$ & $\vec{n}_{2}$ & $\vec{u}$ \\
\hline \hline$\vec{n}_{1}$ & $\overrightarrow{0}$ & $\overrightarrow{0}$ & $\vec{n}_{1}$ \\
\hline$\vec{n}_{2}$ & $\overrightarrow{0}$ & $\overrightarrow{0}$ & $\vec{u}$ \\
\hline$\vec{u}$ & $\vec{n}_{1}$ & $\vec{u}$ & $\vec{u}+\vec{n}_{1}-\vec{n}_{2}$ \\
\hline
\end{tabular}


or, equivalently, the quadratic system

$$
\begin{aligned}
& \dot{x}=2 x z+z^{2}, \\
& \dot{y}=-z^{2}, \\
& \dot{z}=z^{2}+2 y z .
\end{aligned}
$$

Then the origin, which is clearly an element of $\mathcal{N}=\operatorname{span}\left(\vec{n}_{1}, \vec{n}_{2}\right)$ is a (Lyapunov) stable singular point of this system, despite the fact that $\vec{u}$ is a nearidempotent associated with $\mathcal{N}$.

Proof. One way to prove that the origin is a stable singular point of the homogeneous system $(\dot{x}, \dot{y}, \dot{z})=\vec{f}(x, y, z)$ is to show that given an initial condition $\left(x_{0}, y_{0}, z_{0}\right)$ there exist some positive real constants $M_{x}, M_{y}$ and $M_{z}$ such that $|x(t)| \leq M_{x}\left|x_{0}\right|,|y(t)| \leq M_{y}\left|y_{0}\right|$ and $|z(t)| \leq M_{z}\left|z_{0}\right|$.

We can reduce the number of terms on the right hand side of (3.4) from 5 to 4 if we apply a simple linear transformation

$$
X=x+\frac{1}{2} y+\frac{1}{2} z, Y=y, Z=z .
$$

Linear transformation in finite dimensions are bounded, therefore the (non)stability of origin is not affected. In this way system (3.4) simplifies to

$$
\begin{aligned}
& \dot{X}=2 X Z, \\
& \dot{Y}=-Z^{2}, \\
& \dot{Z}=2 Y Z+Z^{2} .
\end{aligned}
$$

In order to estimate the growth of coordinate $X$, we introduce cylindrical-type coordinates

$$
\begin{aligned}
Y(t) & =R(t) \cos \varphi(t), \\
Z(t) & =R(t) \sin \varphi(t) .
\end{aligned}
$$

From $\varphi=\arctan (Z / Y)$ it follows

$$
\frac{d \varphi}{d t}=\frac{\dot{Z} Y-\dot{Y} Z}{Y^{2}+Z^{2}}
$$

which further implies

$$
\begin{aligned}
\frac{d X}{d \varphi} & =\frac{d X}{d t} \cdot \frac{d t}{d \varphi}=2 X Z \frac{Y^{2}+Z^{2}}{\dot{Z} Y-\dot{Y} Z} \\
& =2 R \sin \varphi \cdot X \cdot \frac{R^{2}}{R^{3}\left(2 \cos ^{2} \varphi \sin \varphi+\sin ^{2} \varphi \cos \varphi+\sin ^{3} \varphi\right)} \\
& =\frac{2}{2 \cos ^{2} \varphi+\sin \varphi \cos \varphi+\sin ^{2} \varphi} X=F(\varphi) X .
\end{aligned}
$$


The function $F(\varphi)$ has no poles and is therefore bounded, for example $F(\varphi)<$ 3 for all $\varphi$. Moreover,

$$
\begin{aligned}
& \int \frac{2 d \varphi}{2 \cos ^{2} \varphi+\sin \varphi \cos \varphi+\sin ^{2} \varphi} \\
& =\frac{4}{\sqrt{7}} \arctan \frac{\sqrt{2}+3 \tan \left(\frac{\pi}{8}+\varphi\right)}{\sqrt{7}} .
\end{aligned}
$$

implies

$$
X(\varphi)=X_{0} e^{\frac{4}{\sqrt{7}} \arctan \frac{\sqrt{2}+3 \tan \left(\frac{\pi}{8}+\varphi\right)}{\sqrt{7}}} \leq e^{\frac{2 \pi}{\sqrt{7}}} \cdot X_{0}
$$

Now it remains to be seen that coordinates $Y$ and $Z$, which form a 2dimensional sub-system

$$
\begin{aligned}
& \dot{Y}=-Z^{2}, \\
& \dot{Z}=2 Y Z+Z^{2}
\end{aligned}
$$

also stay bounded near the origin. First we compute

$$
\begin{aligned}
\frac{d R}{d \varphi} & =\frac{R^{2}(\sin \varphi+\cos \varphi) \sin ^{2} \varphi}{R\left(2 \cos ^{2} \varphi+\cos \varphi \sin \varphi+\sin ^{2} \varphi\right) \sin \varphi} \\
& =R \frac{(\sin \varphi+\cos \varphi) \sin \varphi}{2 \cos ^{2} \varphi+\cos \varphi \sin \varphi+\sin ^{2} \varphi}=R \cdot G(\varphi) .
\end{aligned}
$$

The function in the denominator has no zeros, therefore $G(\varphi)$ is a bounded function defined everywhere on the real axis, so we can integrate

$$
\begin{aligned}
& \int \frac{(\sin \varphi+\cos \varphi) \sin \varphi d \varphi}{2 \cos ^{2} \varphi+\cos \varphi \sin \varphi+\sin ^{2} \varphi} \\
& \quad=\ln \sqrt{\frac{1}{2 \cos ^{2} \varphi+\cos \varphi \sin \varphi+\sin ^{2} \varphi}}+\frac{1}{\sqrt{7}} \arctan \frac{\sqrt{2}+3 \tan \left(\frac{\pi}{8}+\varphi\right)}{\sqrt{7}} \\
& \quad=H(\varphi) .
\end{aligned}
$$

Since $R(\varphi)=R_{0} e^{H(\varphi)}$, it follows

$$
R(\varphi) \leq \max \sqrt{\frac{1}{2 \cos ^{2} \varphi+\cos \varphi \sin \varphi+\sin ^{2} \varphi}} e^{\frac{\pi}{2 \sqrt{7}}} R_{0}<\sqrt{2} e^{\frac{\pi}{2 \sqrt{7}}} R_{0} .
$$

If $(X, Y, Z)$ is a solution of $(3.5)$ with initial condition $\left(X_{0}, Y_{0}, Z_{0}\right)$ which lies inside of some $\varepsilon-$ neighborhood of the origin $(0,0,0)$, we have

$$
X_{0}^{2}+Y_{0}^{2}+Z_{0}^{2}<\varepsilon^{2},
$$


while both estimates completed above imply that the solution satisfies

$$
\begin{aligned}
X^{2}+Y^{2}+Z^{2} & =X^{2}+R^{2}<e^{\frac{4 \pi}{\sqrt{7}}} \cdot X_{0}^{2}+2 e^{\frac{\pi}{\sqrt{7}}} R_{0}^{2} \\
& =e^{\frac{4 \pi}{\sqrt{7}}} \cdot X_{0}^{2}+2 e^{\frac{\pi}{\sqrt{7}}}\left(Y_{0}^{2}+Z_{0}^{2}\right) \\
& <e^{\frac{4 \pi}{\sqrt{7}}} X_{0}^{2}+e^{\frac{4 \pi}{\sqrt{7}}}\left(Y_{0}^{2}+Z_{0}^{2}\right) \\
& <e^{\frac{4 \pi}{\sqrt{7}}} \varepsilon^{2}
\end{aligned}
$$

and therefore remains inside $e^{\frac{2 \pi}{\sqrt{7}}} \varepsilon-$ neighborhood of the origin. This means that $\vec{N}=\overrightarrow{0}$ (i.e. $X=Y=Z=0$ ) is a stable singular point of $X, Y, Z-$ system. Since this system is linearly equivalent to $(x, y, z)-$ system (3.4), it follows that $\vec{n}=\overrightarrow{0}$ (i.e. $x=y=z=0$ ) is a stable nilpotent in $\mathcal{N}$.

\section{ACKNOWLEDGEMENTS.}

The authors acknowledge the financial support from the Slovenian Research Agency (research core funding No. P1-0288) and the project Algebraic Methods for the Application of Differential Equations No. N1-0063.

The authors thank both referees for their careful reading and helpful comments, which improved the presentation of the paper.

\section{REFERENCES}

[1] J. Argemi, Sur les points singuliers multiples de systèmes dynamiques dans $\mathbb{R}^{2}$, Ann. Mat. Pura Appl. (4) 79 (1968), 35-69.

[2] Z. Balanov and Y. Krasnov, Complex structures in algebra, topology and differential equations, Georgian Math. J. 21 (2014), 249-260.

[3] H. Boujemaa, M. Rachidi and A. Micali, On a class of nonassociative algebras: a reduction theorem for their associated quadratic systems, Algebras Groups Geom. 19 (2002), 73-83.

[4] H. Boujemaa, S. El Qotbi, H. Rouiouih, Stability of critical points of quadratic homogeneous dynamical systems, Glas. Mat. Ser. III 51(71) (2016), 165-173.

[5] I. Burdujan, A class of commutative algebras and their applications in Lie triple system theory, ROMAI J. 3 (2007), 15-39.

[6] I. Burdujan, Automorphisms and derivations of homogeneous quadratic differential systems, ROMAI J. 6 (2010) 15-28.

[7] C. B. Collins, Algebraic classification of homogeneous polynomial vector fields in the plane, Japan J. Indust. Appl. Math. 13 (1996), 63-91.

[8] C. B. Collins, Two-dimensional homogeneous polynomial vector fields with common factors, J. Math. Anal. Appl. 181 (1994), 836-863.

[9] T. Date and M. Iri, Canonical forms of real homogeneous quadratic transformations, J. Math. Anal. Appl. 56 (1976), 650-682.

[10] J. L. Kaplan and J. A. Yorke, Nonassociative, real algebras and quadratic differential equations, Nonlinear Anal. 3 (1979), 49-51.

[11] M. K. Kinyon and A. A. Sagle, Differential systems and algebras, in Differential equations, dynamical systems, and control science, Dekker NY, 1994, 115-141.

[12] M. K. Kinyon and A. A. Sagle, Quadratic dynamical systems and algebras, J. Differential Equations 117 (1995), 67-126. 
[13] Y. Krasnov, Properties of ODEs and PDEs in algebras, Complex Anal. Oper. Theory 7 (2013), 623-634.

[14] Y. Krasnov and I. Messika, Differential and integral equations in algebra, Funct. Differ. Equ. 21 (2014), 137-146.

[15] M. Kutnjak and M. Mencinger, A family of completely periodic quadratic discrete dynamical system, Internat. J. Bifur. Chaos Appl. Sci. Engrg. 18 (2008), 1425-1433.

[16] L. Lyagina, The integral curves of the equation $y^{\prime}=\left(a x^{2}+b x y+c y^{2}\right) /\left(d x^{2}+e x y+\right.$ $\left.f y^{2}\right)$, (Russian) Uspehi Matem. Nauk (N.S.) 6 (1951), 171-183.

[17] L. Markus, Quadratic differential equations and non-associative algebras, in Contributions to the theory of nonlinear oscillations, 1960, 185-213.

[18] N. Mehdi and M. Mohsen, Classification the integral curves of a second degree homogeneous ODE, Math. Sci. Q. J. 4 (2010), 371-381.

[19] M. Mencinger, On stability of the origin in quadratic systems of ODEs via Markus approach, Nonlinearity 16 (2003) 201-218.

[20] M. Mencinger, Stability analysis of critical points in quadratic systems in $\mathbb{R}^{3}$ which contain a plane of critical points, in Let's face chaos through nonlinear dynamics (Maribor, 2002), Progr. Theoret. Phys. Suppl. 150 (2003), 388-392.

[21] M. Mencinger, On stability of Riccati differential equation $\dot{X}=T X+Q(X)$ in $\mathbb{R}^{n}$, Proc. Edinb. Math. Soc. (2) 45 (2002), 601-615.

[22] M. Mencinger and M.Kutnjak, The dynamics of NQ-systems in the plane, Internat. J. Bifur. Chaos Appl. Sci. Engrg. 19 (2009), 117-133.

[23] M. Mencinger and B. Zalar, On stability of critical points of quadratic differential equations in nonassociative algebras, Glas. Mat. Ser. III 38(58) (2003), 19-27.

[24] M. Mencinger, B. Zalar, A class of nonassociative algebras arising from quadratic ODEs, Comm. Algebra. 33 (2005), 807-828.

[25] H. C. Myung and A. A. Sagle, Quadratic differential equations and algebras, Contemp. Math. 131, AMS, Providence, 1992, 659-672.

[26] S. Walcher, Algebras and differential equations, Hadronic Press, Inc., Palm Harbor, 1991.

B. Zalar

Faculty of Civil Engineering, Transportation Engineering and Architecture

University of Maribor

Smetanova 17, 2000 Maribor

Slovenia

E-mail: borut.zalar@um.si

M. Meninger

Faculty of Civil Engineering,Transportation Engineering and Architecture

University of Maribor

Smetanova 17, 2000 Maribor

and

Institute of Mathematics, Physics and Mechanics

Jadranska 19, 1000 Ljubljana

and

Center for Applied Mathematics and Theoretical Physics

University of Maribor

Mladinska 3, 2000 Maribor

Slovenia

E-mail: matej.mencinger@um.si

Received: 8.10.2018. 